\title{
Pacific
}

Journal of

Mathematics

\section{MÖBIUS-INVARIANT HILBERT SPACES IN POLYDISCS}

H. TURGAY KAPTANOGLU

Volume 163 No. 2

April 1994 


\title{
MÖBIUS-INVARIANT HILBERT SPACES IN POLYDISCS
}

\section{H. Turgay KaptanoĞLU}

\begin{abstract}
We define the Dirichlet space $\mathscr{D}$ on the unit polydisc $\mathbb{U}^{n}$ of $\mathbb{C}^{n} . \mathscr{D}$ is a semi-Hilbert space of of holomorphic functions, contains the holomorphic polynomials densely, is invariant under compositions with the biholomorphic automorphisms of $\mathbb{U}^{n}$, and its semi-norm is preserved under such compositions. We show that $\mathscr{D}$ is unique with these properties. We also prove $\mathscr{D}$ is unique if we assume that the semi-norm of a function in $\mathscr{D}$ composed with an automorphism is only equivalent in the metric sense to the semi-norm of the original function. Members of a subclass of $\mathscr{D}$ given by a norm can be written as potentials of $\mathscr{L}^{2}$-functions on the $n$-torus $\mathbb{T}^{n}$. We prove that the functions in this subclass satisfy strong-type inequalities and have tangential limits almost everywhere on $\partial \mathbb{U}^{n}$. We also make capacitory estimates on the size of the exceptional sets on $\partial \mathbb{U}^{n}$.
\end{abstract}

1. Introduction. Möbius-invariant spaces. Let $\mathbb{U}$ be the open unit disc in $\mathbb{C}$ and $\mathbb{T}$ be the unit circle bounding it. The open unit polydisc $\mathbb{U}^{n}$ and the torus $\mathbb{T}^{n}$ in $\mathbb{C}^{n}$ are the cartesian products of $n$ unit discs and $n$ unit circles, respectively. $\mathbb{T}^{n}$ is the distinguished boundary of $\mathbb{U}^{n}$ and forms only a small part of the topological boundary $\partial \mathbb{U}^{n}$ of $\mathbb{U}^{n}$. We denote by $\mathscr{M}$ the group of all biholomorphic automorphisms of $\mathbb{U}^{n}$ (the Möbius group). The subgroup of linear automorphisms in $\mathscr{M}$ is denoted by $\mathscr{U}$. The space of holomorphic functions with domain $\mathbb{U}^{n}$ will be called $\mathscr{H}\left(\mathbb{U}^{n}\right)$ and will carry the topology of uniform convergence on compact subsets of $\mathbb{U}^{n}$.

A semi-inner product on a complex vector space $\mathscr{H}$ is a sesquilinear functional on $\mathscr{H} \times \mathscr{H}$ with all the properties of an inner product except that it is possible to have $\langle\langle a, a\rangle\rangle=0$ when $a \neq 0$. $\|a\|=\sqrt{\langle\langle a, a\rangle\rangle}$ is the associated semi-norm. We assume $\langle\langle\cdot, \cdot\rangle\rangle$ is not identically zero.

DEFINITION 1.1. $\mathscr{H}$ is called a Hilbert space of holomorphic functions on $\mathbb{U}^{n}$ if

(i) $\mathscr{H}$ is a linear subspace of $\mathscr{H}\left(\mathbb{U}^{n}\right)$,

(ii) the semi-inner product $\langle\langle\cdot, \cdot\rangle$ of $\mathscr{H}$ is complete,

(iii) $\mathscr{H}$ contains all (holomorphic) polynomials,

(iv) polynomials are dense in $\mathscr{H}$ in the topology of the semi-norm $\|\cdot\|$ of $\mathscr{H}$. 
A space $\mathscr{H}$ of functions on $\mathbb{U}^{n}$ is $\mathscr{M}$-invariant if $f \circ \Psi \in \mathscr{H}$ whenever $f \in \mathscr{H}$ and $\Psi \in \mathscr{M}$. An $\mathscr{M}$-invariant Hilbert space $\mathscr{H}$ of holomorphic functions on $\mathbb{U}^{n}$ will be called an $\mathscr{M}$-space for brevity. $\mathscr{U}$-invariance and $\mathscr{U}$-space have similar definitions.

$\mathbb{N}, \mathbb{Z}_{+}, \mathbb{Z}, \mathbb{R}$ denote the set of nonnegative integers, positive integers, integers, and real numbers, respectively. A multi-index $\alpha=$ $\left(\alpha_{1}, \ldots, \alpha_{n}\right)$ is a point in $\mathbb{N}^{n} . \sum_{\alpha}$ indicates a summation with $\alpha$ running over all the points in $\mathbb{N}^{n}$, and $\sum_{\alpha \in \mathbb{I}}^{\prime}$ is a summation where we consider only those $\alpha$ in the index set $\mathbb{I}$ with all positive components. Let also $D_{j}=\partial / \partial z_{j}$ and $\bar{D}_{j}=\partial / \partial \bar{z}_{j}$. The following abbreviated notations will be used:

$$
\begin{aligned}
|\alpha| & =\alpha_{1}+\cdots+\alpha_{n}, & z^{\alpha} & =z_{1}^{\alpha_{1}} \cdots z_{n}^{\alpha_{n}}, \\
\alpha ! & =\alpha_{1} ! \cdots \alpha_{n} !, & D^{\alpha} & =D_{1}^{\alpha_{1}} \cdots D_{n}^{\alpha_{n}} .
\end{aligned}
$$

The Dirichlet space $\mathscr{D}\left(\mathbb{U}^{n}\right)$ is the class of $f(z)=\sum_{\alpha} f_{\alpha} z^{\alpha} \in \mathscr{H}\left(\mathbb{U}^{n}\right)$ with

$$
\|f\|_{\mathscr{D}}^{2}=\sum_{\alpha} \alpha_{1} \cdots \alpha_{n}\left|f_{\alpha}\right|^{2}=\sum_{k=0}^{\infty} \sum_{|\alpha|=k} \alpha_{1} \cdots \alpha_{n}\left|f_{\alpha}\right|^{2}<\infty .
$$

Equivalently, $\mathscr{D}\left(\mathbb{U}^{n}\right)$ is the class of those $f \in \mathscr{H}\left(\mathbb{U}^{n}\right)$ with

$$
\|f\|_{\mathscr{D}}^{2}=\int_{\mathbb{U}^{n}}\left|D_{1} \cdots D_{n} f\right|^{2} d \mu_{n}<\infty
$$

where $\mu_{n}$ is the Lebesgue maesure on $\mathbb{U}^{n}$ normalized so that $\mu_{n}\left(\mathbb{U}^{n}\right)=$ 1. The semi-norm $\|\cdot\|_{\mathscr{D}}$ is obtained from the semi-inner product

$$
\left\langle\langle f, g\rangle_{\mathscr{D}}=\sum_{\alpha} \alpha_{1} \cdots \alpha_{n} f_{\alpha} \bar{g}_{\alpha}=\int_{\mathbb{U}^{n}}\left(D_{1} \cdots D_{n} f\right) \overline{\left(D_{1} \cdots D_{n} g\right)} d \mu_{n} .\right.
$$

Main results. In this work, we first prove two theorems which show that the Dirichlet space is unique among $\mathscr{M}$-spaces that have certain properties.

Theorem A. Let $\mathscr{H}$ be an $\mathscr{M}$-space and suppose that

$$
\|f\|=\|f \circ \Psi\| \quad(f \in \mathscr{H}, \Psi \in \mathscr{M}) .
$$

Then

$$
\|f\|=C\|f\|_{\mathscr{D}} \quad(f \in \mathscr{H}),
$$

where $C=\left\|z_{1} \cdots z_{n}\right\|^{2}$. Thus $\mathscr{H}$ is $\mathscr{D}\left(\mathbb{U}^{n}\right)$. 
Note that the assumption on the semi-norm is equivalent to $\langle\langle f \circ \Psi, g \circ \Psi\rangle\rangle=\langle\langle f, g\rangle$, and the conclusion implies that $\langle\langle f, g\rangle\rangle=C\left\langle\langle f, g\rangle_{\mathscr{D}}\right.$, for all $f, g \in \mathscr{H}$ and $\Psi \in \mathscr{M}$.

For the second theorem, we need a strengthening of condition (ii) of Definition 1.1. To derive it, we write the Taylor series expansion at 0 of an $f \in \mathscr{H}$ by seperating the higher and lower dimensional terms. For example, when $n=2$, using $(z, w)$ for $\left(z_{1}, z_{2}\right)$ and $(k, l)$ for $\left(\alpha_{1}, \alpha_{2}\right)$, we write

$$
f(z, w)=f_{00}+\sum_{k=1}^{\infty} f_{k 0} z^{k}+\sum_{l=1}^{\infty} f_{0 l} w^{l}+\sum_{k, l=1}^{\infty} f_{k l} z^{k} w^{l} .
$$

Since we assume conditions (iii) and (iv) of Definition 1.1, we can define a norm on $\mathscr{H}$ by

$\left|\left\|f\left|\left\|^{2}=\left|f_{00}\right|^{2}+\sum_{k=1}^{\infty}\left|f_{k 0}\right|^{2}\right\| z^{k}\left\|_{1}^{2}+\sum_{l=1}^{\infty}\left|f_{0 l}\right|^{2}\right\| w^{l}\left\|_{1}^{2}+\sum_{k, l=1}^{\infty}\left|f_{k l}\right|^{2}\right\| z^{k} w^{l} \|^{2}\right.\right.\right.$,

where $\|\cdot\|$ is still the semi-norm of $\mathscr{H} \subset \mathscr{H}\left(\mathbb{U}^{n}\right)$ and $\|\cdot\|_{1}$ denotes the semi-norm of a similar $\mathscr{H}^{\prime} \subset \mathscr{H}(\mathbb{U})$. We already know (see [1]) that $\|\cdot\|_{1}$ is equvalent to $\|\cdot\|_{\mathscr{D}}$ in $\mathbb{U}$. Since our proof of Theorem B is by induction on the dimension of the polydisc, the above definition of ||$|\cdot|||$ makes sense. Now we make the alternate assumption

(ii) $^{\prime} \mathscr{H}$ is complete in the norm ||$|\cdot| \mid$.

A similar condition was assumed in [1], whereas [4] assumes (ii).

THEOREM B. Let $\mathscr{H}$ be an $\mathscr{M}$-space in the sense modified by (ii)' and assume that there is a positive constant $\delta<1$ such that

$$
\delta\|f\| \leq\|f \circ \Psi\| \leq \frac{1}{\delta}\|f\| \quad(f \in \mathscr{H}, \Psi \in \mathscr{M}) .
$$

Then there exists positive constants $K_{1}$ and $K_{2}$ such that

$$
K_{1}\|f\|_{\mathscr{D}} \leq\|f\| \leq K_{2}\|f\|_{\mathscr{D}} \quad(f \in \mathscr{H}) .
$$

Thus $\mathscr{D}\left(\mathbb{U}^{n}\right)$ is unique again.

The proofs of these theorems will be presented in $\S 2$.

Next, we consider a subspace of the Dirichlet space, one that is defined by a genuine norm similar to ||$\cdot \mid \|$. This space is not $\mathscr{M}$ invariant any more, but the stronger conditions on it allow us to prove 
that it has tangential limits as we approach $\partial \mathbb{U}^{n}$. In fact, tangential limits exist for a wider class of functions which are potentials of certain functions in $\mathscr{L}^{2}\left(\mathbb{T}^{n}\right)$. The precise definitions and theorems are stated in $\S 3$. Theorems $C$ and $D$ at the end of that section are the major results in this direction.

In earlier work, Arazy and Fisher [1] proved, under slightly different hypotheses, the analogs of Theorem A and Theorem B in $\mathbb{U}$. Zhu [5] found the equivalent of Theorem $A$ for the unit ball in $\mathbb{C}^{n}$ when $n \geq 2$. Nagel, Rudin and Shapiro [3] obtained the unit-disc versions of Theorems $\mathrm{C}$ and $\mathrm{D}$.

After the submission of the manuscript, we were informed by a referee that in the preprint Invariant Hilbert Spaces of Analytic Functions on Bounded Symmetric Domains by J. Arazy and S. D. Fisher, results analogous to Theorems A and B were established for all irreducible bounded symmetric domains.

Notation. $\lambda_{n}$ is the Lebesgue measure on $\mathbb{T}^{n}$ both normalized to have mass 1 ; i.e., it is the Haar measure on the compact abelian group $\mathbb{T}^{n}$. If $p \in[1, \infty)$, its conjugate is $q=p /(p-1)$. The $\mathscr{L}^{p}$ - and $\ell^{p}$ spaces will have their usual meaning. $z_{j}$ will usually be an element of $\mathbb{U}$ and $\zeta_{j}$ of $\mathbb{T}$. Apart from the usual big $\mathscr{O}$ notation, we will use $u \sim v$ to mean both $u=\mathscr{O}(v)$ and $v=\mathscr{O}(u)$, and $u \approx v$ to mean $u / v$ has a finite positive limit.

The Poisson integral of an $f \in \mathscr{L}^{1}\left(\mathbb{T}^{n}\right)$ is

$$
P[f](z)=\int_{\mathbb{T}^{n}} f(\zeta) \prod_{j=1}^{n} \frac{1-\left|z_{j}\right|^{2}}{\left|1-z_{j} \bar{\zeta}_{j}\right|^{2}} d \lambda_{n}(\zeta) \quad\left(z \in \mathbb{U}^{n}\right),
$$

and its Cauchy integral is

$$
C[f](z)=\int_{\mathbb{T}^{n}} f(\zeta) \prod_{j=1}^{n} \frac{1}{1-z_{j} \bar{\zeta}_{j}} d \lambda_{n}(\zeta) \quad\left(z \in \mathbb{U}^{n}\right),
$$

where the products are called the Poisson kernel $P(z, \zeta)$ and the Cauchy kernel $C(z, \zeta)$ for $\mathbb{U}^{n}$, respectively. These transforms have the following invariance properties: If $f \in \mathscr{L}^{1}\left(\lambda_{n}\right), \Psi \in \mathscr{M}$, and $U \in \mathscr{U}$, then

$$
P[f \circ \Psi]=P[f] \circ \Psi \quad \text { and } \quad C[f \circ U]=C[f] \circ U .
$$

The automorphisms of $\mathbb{U}^{n}$ for $n \geq 2$ are generated by the following three subgroups: rotations in each variable separately

$$
R_{\theta}(z)=\left(e^{i \theta_{1}} z_{1}, \ldots, e^{i \theta_{n}} z_{n}\right),
$$


Möbius transformations in each variable separately

$$
\Phi_{w}(z)=\left(\phi_{w_{1}}\left(z_{1}\right), \ldots, \phi_{w_{n}}\left(z_{n}\right)\right),
$$

and the coordinate permutations. Here $\theta \in[-\pi, \pi]^{n}$ and $w \in \mathbb{U}^{n}$ are fixed, Möbius transformations are in the form

$$
\phi_{w}(z)=\frac{w-z}{1-\bar{w} z} \quad(w \in \mathbb{U}, \quad z \in \overline{\mathbb{U}}),
$$

and the coordinate permutations are nothing but the $n$ ! members of the symmetric group $\mathscr{S}_{n}$ on $n$ objects. Thus an arbitrary $\Psi \in \mathscr{M}$ can be written in the form

$$
\Psi(z)=\left(e^{i \theta_{1}} \phi_{w_{1}}\left(z_{\sigma(1)}\right), \ldots, e^{i \theta_{n}} \phi_{w_{n}}\left(z_{\sigma(n)}\right)\right),
$$

for some $w \in \mathbb{U}^{n}$ and $\theta \in[-\pi, \pi]^{n}$, and $\sigma \in \mathscr{S}_{n}$. $\mathscr{U}$ is generated by $\sigma \in \mathscr{S}_{n}$ and the rotations $R_{\theta}$. Each Möbius transformation $\Phi_{w}$ is an involution (its inverse is itself) exchanging 0 and $w$. $\mathscr{M}$ acts transitively on $\mathbb{U}^{n}:$ if $a, b \in \mathbb{U}^{n}$, then $\Phi_{b} \circ \Phi_{a} \in \mathscr{M}$ moves $a$ to $b$ (and $b$ to $a$ ). Finally, $\mathscr{M}^{*}$ denotes the component of the identity in $\mathscr{M}$; i.e., $\mathscr{M}^{*}$ is $\mathscr{M}$ without the action of $\mathscr{S}_{n}$.

2. Uniqueness of the Dirichlet space. We start by showing that $\mathscr{D}\left(\mathbb{U}^{n}\right)$ has all the properties of a Hilbert space in the sense of Definition 1.1. Clearly the polynomials are in $\mathscr{D}\left(\mathbb{U}^{n}\right)$ and $\left\|z^{\alpha}\right\|^{2}=\alpha_{1} \cdots \alpha_{n}$ for all $\alpha \in \mathbb{N}^{n}$. A quick look at (1.1) shows that the polynomials are dense in $\mathscr{D}\left(\mathbb{U}^{n}\right)$ with respect to $\|\cdot\|_{\mathscr{D}}$. Again from (1.1), identifying $g$ by $\left\{g_{\alpha}\right\}$, we see that $\mathscr{D}\left(\mathbb{U}^{n}\right)$ is a weighted $\ell^{2}$-space, hence every Cauchy sequence $\left\{f_{j}\right\}$ in $\mathscr{D}\left(\mathbb{U}^{n}\right)$ converges in $\|\cdot\|_{\mathscr{D}}$ to some $f \in \mathscr{D}\left(\mathbb{U}^{n}\right)$ represented by $\left\{f_{\alpha}\right\}$ for $\alpha \in \mathbb{Z}_{+}^{n}$. To show that $f$ is holomorphic, let $f_{m}(z)=\sum_{k=1}^{m} \sum_{|\alpha|=k}^{\prime} f_{\alpha} z^{\alpha}$ and pick $\varepsilon>0$. For any $0<r<1$ and positive integers $m>l>n$,

$$
\begin{aligned}
\sup _{z \in r \overline{\mathbb{U}}^{n}}\left|\left(f_{m}-f_{l}\right)(z)\right| & =\sup _{z \in r \overline{\mathbb{U}}^{n}}\left|\sum_{k=l+1}^{m} \sum_{|\alpha|=k}^{\prime} f_{\alpha} z^{\alpha}\right| \leq \sum_{k=l+1}^{m} \sum_{|\alpha|=k}^{\prime}\left|f_{\alpha}\right| r^{|\alpha|} \\
& \leq\left(\sum_{k=l+1}^{m} \sum_{|\alpha|=k}^{\prime}\left|f_{\alpha}\right|^{2}\right)^{1 / 2}\left(\sum_{k=l+1}^{m} \sum_{|\alpha|=k}^{\prime} r^{2|\alpha|}\right)^{1 / 2} \\
& <\left(\sum_{k=l+1}^{m} \sum_{|\alpha|=k} \alpha_{1} \cdots \alpha_{n}\left|f_{\alpha}\right|^{2}\right)^{1 / 2}\left(\sum_{k=l+1}^{m} k^{n} r^{2 k}\right)^{1 / 2} .
\end{aligned}
$$

The first factor is less than $\varepsilon$ when $l$ and $m$ are large enough because $f \in \mathscr{D}\left(\mathbb{U}^{n}\right)$, and the second factor is bounded as $l, m \rightarrow \infty$. Hence 
$f(z)=\sum_{\alpha}^{\prime} f_{\alpha} z^{\alpha}$ is uniformly convergent on compact subsets of $\mathbb{U}^{n}$, and this proves $f \in \mathscr{H}\left(\mathbb{U}^{n}\right)$. Note that we need not know $f_{\alpha}$ if $\alpha \in \mathbb{N}^{n} \backslash \mathbb{Z}_{+}^{n}$. These coefficients of $f$ can be taken arbitrarily as long as $f$ remains holomorphic.

LEMMA 2.1. $\mathscr{M}$ is generated by $\mathscr{S}_{n}$, rotations $R_{\omega}(z)=\left(e^{i \omega} z_{1}, z_{2}\right.$, $\left.\ldots, z_{n}\right)$ with $\omega \in[-\pi, \pi]$, and Möbius transformations of the form $\Phi_{t}(z)=\left(\phi_{t}\left(z_{1}\right), z_{2}, \ldots, z_{n}\right)$ with $0 \leq t<1$.

Proposition 2.2. $\mathscr{D}\left(\mathbb{U}^{n}\right)$ is $\mathscr{M}$-invariant.

Proof. The integral form of the Dirichlet semi-norm uses the measure $\mu_{n}$ which is invariant under rotations and permutations. Thus $\mathscr{D}\left(\mathbb{U}^{n}\right)$ is $\mathscr{U}$-invariant. To prove invariance under Möbius transformations, in view of Lemma 2.1, it suffices to consider

$$
w=\Phi_{r}(z)=\left(\frac{r-z_{1}}{1-r z_{1}}, z_{2}, \ldots, z_{n}\right)
$$

Then $D_{1}^{z}\left(f \circ \Phi_{r}\right)=\left(D_{1}^{w} f\right) d w_{1} / d z_{1}$ and

$$
\begin{aligned}
& \left|D_{1}^{z} D_{2} \cdots D_{n}\left(f \circ \Phi_{r}\right)\right|^{2}=\left|D_{1}^{w} D_{2} \cdots D_{n} f\right|^{2} \frac{\left(r^{2}-1\right)^{2}}{\left|1-r z_{1}\right|^{4}} \\
& =\left|D_{1}^{w} D_{2} \cdots D_{n} f\right|^{2} J_{\Re} \Phi_{r}(z),
\end{aligned}
$$

since $d w_{1} / d z_{1}=\left(r^{2}-1\right) /\left(1-r z_{1}\right)^{2}$, where $J_{\Re} \Phi_{r}$ is the real Jacobian of $\Phi_{r}$. Therefore

$$
\begin{aligned}
\left\|f \circ \Phi_{r}\right\|_{\mathscr{D}}^{2} & =\int_{\mathbb{U}^{n}}\left|D_{1}^{z} D_{2} \cdots D_{n}\left(f \circ \Phi_{r}\right)(z)\right|^{2} d \mu_{n}(z) \\
& =\int_{\mathbb{U}^{n}}\left|D_{1}^{w} D_{2} \cdots D_{n} f(w)\right|^{2} \frac{\left(r^{2}-1\right)^{2}}{\left|1-r z_{1}\right|^{4}} \frac{1}{J_{\Re} \Phi_{r}(z)} d \mu_{n}(w) \\
& =\int_{\mathbb{U}^{n}}\left|D_{1}^{w} D_{2} \cdots D_{n} f(w)\right| d \mu_{n}(w)=\|f\|_{\mathscr{D}}^{2} .
\end{aligned}
$$

Note that when $n \geq 2, \mathscr{D}\left(\mathbb{U}^{n}\right)$ does not put any conditions on the infinitely many power series coefficients of $f$, those with at least one $\alpha_{j}=0$, i.e., those in $\mathbb{N}^{n} \backslash \mathbb{Z}_{+}^{n}$. Thus if each term in the Taylor expansion of some $f \in \mathscr{H}\left(\mathbb{U}^{n}\right)$ depends on fewer than $n$ variables, then $\|f\|_{\mathscr{D}}=0$ and $f \in \mathscr{D}$. The Dirichlet space can also be thought 
of as a quotient space of holomorphic functions satisfying (1.1) where the functions whose Taylor series differ by terms depending on at most $n-1$ variables are identified. Trivially any holomorphic function $f$ of fewer than $n$ variables or any constant $f$ has $\|f\|_{\mathscr{D}}=0$ and is in $\mathscr{D}\left(\mathbb{U}^{n}\right)$. For comparison, when $n=1$, only constants (a onedimensional subspace) have zero Dirichlet semi-norm.

We can define a modified Dirichlet space $\tilde{\mathscr{D}}\left(\mathbb{U}^{n}\right)$ similar to $\mathscr{D}\left(\mathbb{U}^{n}\right)$ by considering a norm instead of a semi-norm. This requires some control on all the power series coefficients of $f \in \mathscr{H}\left(\mathbb{U}^{n}\right)$. For simplicity let's look at the case $n=2$. With notation as before, let

$$
\begin{aligned}
\|f\|_{\tilde{D}}^{2}= & \left|f_{00}\right|^{2}+\sum_{k=1}^{\infty} k\left|f_{k 0}\right|^{2}+\sum_{l=1}^{\infty} l\left|f_{0 l}\right|^{2}+\sum_{k, l=1}^{\infty} k l\left|f_{k l}\right|^{2} \\
= & |f(0,0)|^{2}+\int_{\mathbb{U}}\left|D_{1} f(z, 0)\right|^{2} d \mu_{1}(z) \\
& +\int_{\mathbb{U}}\left|D_{2} f(0, w)\right|^{2} d \mu_{1}(w) \\
& +\int_{\mathbb{U}^{2}}\left|D_{1} D_{2} f(z, w)\right|^{2} d \mu_{2}(z, w) .
\end{aligned}
$$

This norm is $\mathscr{U}$-invariant, but not $\mathscr{M}$-invariant; in fact, none of its first three terms is preserved under compositions with $\Phi_{r}$.

Proof of Theorem A. First, $\left\langle\left\langle z^{\alpha}, z^{\beta}\right\rangle\right\rangle=0$ if $\alpha \neq \beta$. To see this, assume, without loss of generality, $\alpha_{1} \neq \beta_{1}$. Let $\omega$ be an irrational multiple of $\pi$ and consider the rotation $R_{\omega}(z)=\left(e^{i \omega} z_{1}, z_{2}, \ldots, z_{n}\right)$. By the $\mathscr{M}$-invariance of $\langle\langle\cdot, \cdot\rangle$,

$$
\begin{aligned}
\left\langle\left\langle z^{\alpha}, z^{\beta}\right\rangle\right\rangle & =\left\langle\left\langle z^{\alpha} \circ R_{\omega}, z^{\beta} \circ R_{\omega}\right\rangle\right\rangle \\
& =\left\langle\left\langle e^{i \alpha_{1} \omega} z_{1}^{\alpha_{1}} z_{2}^{\alpha_{2}} \cdots z_{n}^{\alpha_{n}}, e^{i \beta_{1} \omega} z_{1}^{\beta_{1}} z_{2}^{\beta_{2}} \cdots z_{n}^{\beta_{n}}\right\rangle\right\rangle \\
& =e^{i\left(\alpha_{1}-\beta_{1}\right) \omega}\left\langle\left\langle z^{\alpha}, z^{\beta}\right\rangle\right\rangle
\end{aligned}
$$

and the desired orthogonality result follows.

Put $C_{\alpha}=\left\langle\left\langle z^{\alpha}, z^{\alpha}\right\rangle\right\rangle$. Note that $C_{\alpha}$ is defined only for $\alpha \in \mathbb{N}^{n}$. If $\beta$ is another multi-index and $\beta=\sigma(\alpha)$ for some $\sigma \in \mathscr{S}_{n}$, then by the $\mathscr{M}$-invariance of $\langle\langle\cdot, \cdot\rangle\rangle$ again, $C_{\alpha}=C_{\beta}$.

Now let $0<r_{j}<1, \Psi=\left(\phi_{r_{1}}, \ldots, \phi_{r_{n}}\right)$, and consider $f(z)=$ $\prod_{j=1}^{n}\left(1-r_{j} z_{j}\right) \in \mathscr{H}$. Then since $z^{\alpha}$ is orthogonal to $z^{\beta}$ for $\alpha \neq \beta$, 
we get

$$
\begin{aligned}
\langle\langle f, f\rangle\rangle= & \left\|\prod_{j=1}^{n}\left(1-r_{j} z_{j}\right)\right\|^{2}=\left\|1-\sum_{j=1}^{n} r_{j} z_{j}+\cdots+(-1)^{m} \prod_{j=1}^{n} r_{j} z_{j}\right\|^{2} \\
= & C_{(0, \ldots, 0)}+\left(\sum_{j=1}^{n} r_{j}^{2}\right) C_{(1,0, \ldots, 0)} \\
& +\left(\sum_{l>j=1}^{n} r_{j}^{2} r_{l}^{2}\right) C_{(1,1,0, \ldots, 0)}+\cdots+\left(\prod_{j=1}^{n} r_{j}^{2}\right) C_{(1, \ldots, 1)}
\end{aligned}
$$

On the other hand,

$$
\begin{aligned}
(f \circ \Psi)(z) & =\prod_{j=1}^{n}\left(1-r_{j} \frac{r_{j}-z_{j}}{1-r_{j} z_{j}}\right)=\prod_{j=1}^{n} \frac{1-r_{j}^{2}}{1-r_{j} z_{j}} \\
& =\prod_{j=1}^{n}\left[\left(1-r_{j}^{2}\right) \sum_{\alpha_{j}=0}^{\infty} r_{j}^{\alpha_{j}} z_{j}^{\alpha_{j}}\right]=\left(\prod_{j=1}^{n}\left(1-r_{j}^{2}\right)\right)\left(\sum_{\alpha} r^{\alpha} z^{\alpha}\right) ;
\end{aligned}
$$

now the density of the polynomials and the axioms of a Hilbert space imply

$$
\langle\langle f \circ \Psi, f \circ \Psi\rangle\rangle=\left(\prod_{j=1}^{n}\left(1-r_{j}^{2}\right)^{2}\right) \sum_{\alpha} r^{2 \alpha} C_{\alpha} .
$$

Putting $x_{j}=r_{j}^{2}$ and using the $\mathscr{M}$-invariance of the semi-norm gives

$$
\begin{array}{r}
\text { (2.1) } C_{(0, \ldots, 0)}+\left(\sum_{j=1}^{n} x_{j}\right) C_{(1,0, \ldots, 0)}+\left(\sum_{l>j=1}^{n} x_{j} x_{l}\right) C_{(1,1,0, \ldots, 0)} \\
+\cdots+\left(\prod_{j=1}^{n} x_{j}\right) C_{(1, \ldots, 1)}=\left(\prod_{j=1}^{n}\left(1-x_{j}\right)^{2}\right) \sum_{\alpha} x^{\alpha} C_{\alpha} .
\end{array}
$$

The constant terms $\left(C_{(0, \ldots, 0)}\right)$ cancel, and if we set the coefficients of $x_{1}, x_{1} x_{2}, \ldots$, and $x_{1} x_{2} \cdots x_{n}$ on either side equal to each other, we obtain, respectively,

$$
\begin{gathered}
C_{(1,0, \ldots, 0)}=C_{(1,0, \ldots, 0)}-2 C_{(0, \ldots, 0)}, \\
C_{(1,1,0, \ldots, 0)}=C_{(1,1,0, \ldots, 0)}-2^{2} C_{(1,0, \ldots, 0)}, \\
\vdots \\
C_{(1, \ldots, 1)}=C_{(1, \ldots, 1)}-2^{n} C_{(1, \ldots, 1,0)} .
\end{gathered}
$$


These imply $C_{(0, \ldots, 0)}=0, C_{(1,0, \ldots, 0)}=0, \ldots, C_{(1, \ldots, 1,0)}=0$. After the elimination of the terms that are zero, (2.1) simplifies to

$$
\begin{aligned}
\sum_{\alpha}^{\prime} x^{\alpha} C_{\alpha} & =\frac{x_{1} \cdots x_{n} C_{(1, \ldots, 1)}}{\left(1-x_{1}\right)^{2} \cdots\left(1-x_{n}\right)^{2}} \\
& =C_{(1, \ldots, 1)}\left(\sum_{\alpha_{1}=1}^{\infty} \alpha_{1} x_{1}^{\alpha_{1}}\right) \cdots\left(\sum_{\alpha_{n}=1}^{\infty} \alpha_{n} x_{n}^{\alpha_{n}}\right) ;
\end{aligned}
$$

and this implies

$$
C_{\alpha}=\alpha_{1} \cdots \alpha_{n} C_{(1, \ldots, 1)} \quad\left(\alpha \in \mathbb{Z}_{+}^{n}\right) .
$$

Thus the norm of a monomial of fewer than $n$ variables is zero. Since the polynomials are dense in $\mathscr{H}$, the same result is true for any $f \in \mathscr{H}$ whose Taylor expansion consists of monomials depending on fewer than $n$ variables. But $C_{(1, \ldots, 1)} \neq 0$, because otherwise, since the polynomials are dense in $\mathscr{H},\langle\cdot, \cdot\rangle\rangle$ would be identically zero contrary to hypothesis. Then renaming $C_{(1, \ldots, 1)}=C$ completes the proof.

Proof of Theorem B. We will only show how the two-variable case is obtained from the one-variable case. This then can be adapted to prove by induction the case for arbitrary $\mathbb{U}^{n}$. Unless explicitly stated, subscripted $C$ 's will denote positive constants that are independent of any parameters.

Step 1 . We begin by introducing two other semi-inner products on $\mathscr{H}$. For $f, g \in \mathscr{H}$, let

$$
[f, g]=\int_{\mathbb{T}^{2}}\left\langle\left\langle f \circ R_{\theta}, g \circ R_{\theta}\right\rangle\right\rangle d \lambda_{2}(\theta)
$$

and

$$
\langle f, g\rangle=m\langle\langle f \circ \Phi, g \circ \Phi\rangle\rangle,
$$

where $m$ is an invariant mean (see [2]) on the abelian subgroup

$$
\mathscr{N}=\left\{\Phi=\left(\phi_{s}, \phi_{t}\right): 0 \leq s, t<1\right\}
$$

of $\mathscr{M}$. To actually make $\mathscr{N}$ abelian, in this proof we change our definition of a Möbius transformation so that $\phi_{w}(z)$ is the negative of what is given in (1.4). The earlier definition was adopted to make $\phi_{w}$ an involution, since it simplified calculations involving $\phi_{w}^{-1}$. The required boundedness condition for the existence of this nonunique 
mean is furnished by (1.3). Rotations and $\mathscr{N}$, along with $\mathscr{S}_{2}$, suffice to generate $\mathscr{M}$, by Lemma 2.1 .

Now $[\cdot, \cdot]$ is rotation-invariant

$$
[f, g]=\left[f \circ R_{\theta}, g \circ R_{\theta}\right] \quad\left(\theta \in[-\pi, \pi]^{n}\right),
$$

and $\langle\cdot, \cdot\rangle$ is $\mathscr{N}$-invariant

$$
\langle f, g\rangle=\langle f \circ \Phi, g \circ \Phi\rangle \quad(\Phi \in \mathscr{N}) .
$$

Moreover, (1.3) implies

$$
\delta^{2}\|f\|^{2} \leq[f, f] \leq \frac{1}{\delta^{2}}\|f\|^{2} \quad(f \in \mathscr{H}),
$$

and

$$
\delta^{2}\|f\|^{2} \leq\langle f, f\rangle \leq \frac{1}{\delta^{2}}\|f\|^{2} \quad(f \in \mathscr{H}) ;
$$

and combining these two, we further obtain

$$
\delta^{4}[f, f] \leq\langle f, f\rangle \leq \frac{1}{\delta^{4}}[f, f] \quad(f \in \mathscr{H}) .
$$

(2.4) and (2.5) show that the semi-norms associated to $[\cdot, \cdot]$ and $\langle\cdot, \cdot\rangle$ are both equivalent to $\|\cdot\|$.

As in the proof of Theorem A, the rotation-invariance of $[\cdot, \cdot]$ gives the orthogonality condition

$$
\left[z^{k_{1}} w^{l_{1}}, z^{k_{2}} w^{l_{2}}\right]=0 \quad\left(\left(k_{1}, l_{1}\right) \neq\left(k_{2}, l_{2}\right)\right),
$$

which leads to

$$
[f, f]=\sum_{k, l=0}^{\infty}\left|f_{k l}\right|^{2}\left[z^{k} w^{l}, z^{k} w^{l}\right] \quad(f \in \mathscr{H}) .
$$

Therefore, to prove the theorem, it suffices to show that

$$
K_{1} k l \leq\left[z^{k} w^{l}, z^{k} w^{l}\right] \leq K_{2} k l \quad\left((k, l) \in \mathbb{N}^{2}\right)
$$

or, equivalently,

$$
K_{3} k l \leq\left\langle z^{k} w^{l}, z^{k} w^{l}\right\rangle \leq K_{4} k l \quad\left((k, l) \in \mathbb{N}^{2}\right)
$$

for some positive constants $K_{1}, K_{2}, K_{3}$, and $K_{4}$. Clearly $K_{2} \geq K_{1}$ and $K_{4} \geq K_{3}$.

Step 2. Claim:

$$
\left\langle z^{k} w^{l}, z^{k} w^{l}\right\rangle \neq 0 \quad \text { if } k \geq 1 \text { and } l \geq 1 .
$$


Suppose it is zero for some $(N, M)$; then $\left[z^{N} w^{M}, z^{N} w^{M}\right]=0$ also. Then for $0 \leq s, t<1$, if we use (2.3), (2.6) and power series expansion

$$
\begin{aligned}
0 & =\left\langle z^{N} w^{M}, z^{N} w^{M}\right\rangle \\
& =\left\langle\left(\frac{z-s}{1-s z}\right)^{N}\left(\frac{w-t}{1-w t}\right)^{M},\left(\frac{z-s}{1-s z}\right)^{N}\left(\frac{w-t}{1-w t}\right)^{M}\right\rangle \\
& \geq \delta^{8}\left[\left(\frac{z-s}{1-s z}\right)^{N}\left(\frac{w-t}{1-w t}\right)^{M},\left(\frac{z-s}{1-s z}\right)^{N}\left(\frac{w-t}{1-w t}\right)^{M}\right] \\
& =\left[\sum_{k=0}^{\infty} c_{k N}(s) z^{k} \sum_{l=0}^{\infty} c_{l M}^{\prime}(t) w^{l}, \sum_{k=0}^{\infty} c_{k N}(s) z^{k} \sum_{l=0}^{\infty} c_{l M}^{\prime}(t) w^{l}\right] \\
& =\sum_{k, l=0}^{\infty}\left|c_{k N}(s)\right|^{2}\left|c_{l M}^{\prime}(t)\right|^{2}\left[z^{k} w^{l}, z^{k} w^{l}\right] .
\end{aligned}
$$

A tedious computation shows that the coefficients $c_{k N}(s) \neq 0$ for any $k, N$, and $s$ as given above; the same is obviously true for $c_{l M}^{\prime}(t)$. Thus $\left[z^{k} w^{l}, z^{k} w^{l}\right]=0$. This means that every element in $\mathscr{H}$ has zero norm and contradicts our basic assumption that $\langle\langle\cdot, \cdot\rangle\rangle$ is not identically zero. Hence the claim is proved.

The one-variable result can be stated as

$$
C_{1} k \leq\left\langle z^{k}, z^{k}\right\rangle_{1} \leq C_{2} k \quad(k \in \mathbb{N}) .
$$

It is a consequence of condition (ii) ${ }^{\prime}$ of Definition 1.1 and of (2.8) that the subspace of $\mathscr{H}$ consisting of functions whose Taylor series expansion at 0 depend only on $z$ is closed. Then (2.9) implies that, for fixed $M \in \mathbb{N}$,

$$
C_{3} k \leq\left\langle z^{k} w^{M}, z^{k} w^{M}\right\rangle \leq C_{4} k \quad(k \in \mathbb{N}),
$$

and we have a similar equation when the power of $z$ is held constant. Of course, the constants $C_{3}$ and $C_{4}$ are different for different $M$. It is our aim to find their explicit dependence on $M$. If we had only finitely many $M$, we could pick $C_{3}$ and $C_{4}$ independently of $M$ and the proof would be over. In the sequel, whenever we have only finitely many $n$ or $M$, we will use this fact without further reference.

Step 3 (upper bound). Let $M \in \mathbb{N}$ be fixed and $k, j \in \mathbb{N}$. Put

$$
\begin{gathered}
\alpha_{k M}^{j M}=\left\langle z^{k} w^{M}, z^{j} w^{M}\right\rangle, \quad \beta_{k M}=\left\langle z^{k} w^{M}, z^{k} w^{M}\right\rangle, \\
b_{k M}=\left[z^{k} w^{M}, z^{k} w^{M}\right] .
\end{gathered}
$$


By (2.3), $\alpha_{k M}^{j M}=\left\langle\phi_{s}^{k} w^{M}, \phi_{s}^{j} w^{M}\right\rangle$ for any $s \in[0,1)$. Differentiate both sides of this equality with respect to $s$ and set $s=0$. Then take $k=j+1$ and add the resulting expressions from $j=0$ to $j=N \geq 1$; and finally divide both sides by $N+1$. The result is

$$
\frac{\beta_{N+1, M}}{N+1}=\frac{2 S_{N M}}{N(N+1)}-\frac{\alpha_{N+2, M}^{N M}}{N},
$$

where $S_{N M}=\frac{1}{2} \beta_{0 M}+\sum_{k=1}^{N} \beta_{k M}$. In particular, $\alpha_{N+2, M}^{N M}$ is real. Now using (2.3) and (2.5), and letting $s^{2}=\frac{N}{N+1}$, we obtain

$$
\begin{aligned}
\frac{1}{\delta^{4}} \beta_{1 M} & =\frac{1}{\delta^{4}}\left\langle\phi_{s} w^{M}, \phi_{s} w^{M}\right\rangle \geq\left[\phi_{s} w^{M}, \phi_{s} w^{M}\right] \\
& =s^{2} b_{0 M}+\left(1-s^{2}\right)^{2} \sum_{k=0}^{\infty} s^{2 k} b_{k+1, M} \\
& \geq \frac{\delta^{4}}{(N+1)^{2}} \sum_{k=0}^{N}\left(\frac{N}{N+1}\right)^{k} \beta_{k+1, M} \geq \frac{\delta^{4}}{(N+1)^{2} e} \sum_{k=0}^{N} \beta_{k+1, M},
\end{aligned}
$$

which implies

$$
S_{N M} \leq\left(\frac{e \beta_{1 M}}{\delta^{8}}+\frac{\beta_{0 M}}{2}\right) N^{2}
$$

Using (2.10) twice on the right side gives

$$
S_{N M} \leq C_{5} M N^{2} \text {. }
$$

It is this inequality and its pair (2.15) below that allow us to pass from one variable to several variables.

As a special case, when $M=0$, we get $S_{N 0}=0$ for all $N \geq 1$. Symmetric nature of the calculation shows also $S_{0 N}=0$ for all $N \geq$ 1. It follows that

$$
\beta_{N 0}=\left\langle z^{N}, z^{N}\right\rangle=0 \text { and } \beta_{0 N}=\left\langle w^{N}, w^{N}\right\rangle=0 \quad\left(N \in \mathbb{Z}_{+}\right) .
$$

The inequality

$$
\delta^{8}\left(\beta_{N M}+\beta_{N+2, M}\right) \leq\left\langle\left(z^{N}+z^{N+2}\right) w^{M},\left(z^{N}+z^{N+2}\right) w^{M}\right\rangle
$$

is a direct consequence of (2.6) and (2.7). Using this, after some routine calculation, $(2.11)$ can be written as

$$
\begin{aligned}
2 \frac{\beta_{N+1, M}}{N+1} & \leq \frac{4 S_{N M}}{N(N+1)}+\frac{2 \beta_{N+2, M}}{N(N+2)}+\left(1-\delta^{8}\right)\left(\frac{\beta_{N M}}{N}+\frac{\beta_{N+2, M}}{N+2}\right) \\
& \leq 6 C_{5} M+\left(1-\delta^{8}\right)\left(\frac{\beta_{N M}}{N}+\frac{\beta_{N+2, M}}{N+2}\right)
\end{aligned}
$$


which is equivalent to $2 \gamma_{N+1, M} \leq\left(1-\delta^{8}\right)\left(\gamma_{N M}+\gamma_{N+2, M}\right)$ if we let

$$
\gamma_{N M}=\frac{\beta_{N M}}{N}-\frac{3 C_{5} M}{\delta^{8}} \text {. }
$$

A result in [1] shows $\gamma_{N+1, M} \leq\left|\gamma_{N M}\right|$ for positive $N$. Then using (2.8), we get

$$
\frac{\beta_{N M}}{N}-\frac{3 C_{5} M}{\delta^{8}} \leq\left|\frac{\beta_{1 M}}{1}-\frac{3 C_{5} M}{\delta^{8}}\right| \leq C_{6} M .
$$

Multiplying both sides by $M$, we conclude

$$
\beta_{N M} \leq\left(C_{6}+\frac{3 C_{5}}{\delta^{8}}\right) N M=K_{4} N M,
$$

which holds for $N \geq 1$ and $M \geq 0$, and for $M \geq 1$ and $N \geq 0$, by the symmetry of the computation.

Step 4 (lower bound). If we combine the result of Step 1 with (2.6), we also get

$$
b_{k M} \leq \frac{K_{4}}{\delta^{4}} k M
$$

We use (2.6), (2.7), (2.3), (2.14), and take $s^{2}=\frac{N}{N+1}$ to calculate

$$
\begin{aligned}
\delta^{8} s^{2} b_{1 M} & \leq \delta^{8}\left[(1+s z) w^{M},(1+s z) w^{M}\right] \\
& =\delta^{4}\left\langle\left(1+s \phi_{s}\right) w^{M},\left(1+s \phi_{s}\right) w^{M}\right\rangle \\
& \leq\left(1-s^{2}\right)^{2}\left[\frac{w^{M}}{1-s z}, \frac{w^{M}}{1-s z}\right]=\left(\frac{1}{N+1}\right)^{2} \sum_{k=0}^{\infty} s^{2 k} b_{k M} \\
& \leq \frac{1}{(N+1)^{2}}\left(\sum_{k=0}^{m N} b_{k M}+\frac{K_{2}}{\delta^{4}} M \sum_{k=m N+1}^{\infty} k\left(\frac{N}{N+1}\right)^{k}\right),
\end{aligned}
$$

where $m$ will be determined shortly. After approximating the second sum by an integral, we have

$$
\delta^{8}\left(\frac{N}{N+1}\right) b_{1 M} \leq \frac{1}{(N+1)^{2}} \sum_{k=0}^{m N} b_{k M}+\frac{K_{2}}{\delta^{4}} M(m+1) e^{-m}
$$

Because of (2.10), $M$ in the last term can be replaced with $C_{8} b_{1 M}$. Now choose $m$ so large that $K_{2} \delta^{-4}(m+1) e^{-m} \leq \delta^{8} / 3$. Using (2.10) once again and some simplification yields

$$
\sum_{k=0}^{N} b_{k M} \geq C_{9} M N^{2}
$$


which is the reverse inequality for (2.12). Combining (2.15) with (2.11), we obtain

$$
\delta^{4} C_{9} M \leq \frac{\beta_{N+1, M}}{N+1}+\left(\frac{\beta_{N M}}{N}\right)^{1 / 2}\left(\frac{\beta_{N+2, M}}{N+2}\right)^{1 / 2} .
$$

Now let

and consider

$$
\phi_{s}^{N}(z)=\left(\frac{z-s}{1-s z}\right)^{N}=\sum_{k=0}^{\infty} c_{k N}(s) z^{k}
$$

$$
\begin{aligned}
b_{N M} & \geq \delta^{4} \beta_{N M}=\delta^{4}\left\langle\phi_{s}^{N} w^{M}, \phi_{s}^{N} w^{M}\right\rangle \geq \delta^{8}\left[\phi_{s}^{N} w^{M}, \phi_{s}^{N} w^{M}\right] \\
& =\delta^{8} \sum_{k=1}^{\infty}\left|c_{k N}(s)\right|^{2} b_{k M} \geq \delta^{8}\left|c_{N+1, N}(s)\right|^{2} b_{N+1, M}
\end{aligned}
$$

A calculation in [1] shows that $\left|c_{N+1, N}(s)\right|^{2} \geq 1 / 2$ for all $s \in[0,1)$ and $N \geq 1$. Thus there is a constant $C_{10}$ such that

$$
\beta_{N+1, M} \leq C_{10} \beta_{N M} \text {. }
$$

Now (2.16) and (2.17) together imply

$$
\beta_{N M} \geq\left(\frac{\delta^{4} C_{9}}{4 C_{10}}\right) N M=K_{3} N M,
$$

for $N \geq 1$ and $M \geq 0$, and for $M \geq 1$ and $N \geq 0$.

Step 5. The only term we have not yet accounted for is $\beta_{00}=\langle 1,1\rangle$. Since it represents a one-dimensional subspace of $\mathscr{H}$, we now know $\mathscr{H}=\mathscr{D}\left(\mathbb{U}^{2}\right)$. To complete the proof, we will also show $\beta_{00}=b_{00}=0$. To obtain a contradiction, suppose $b_{00}=[1,1] \neq 0$. Let $f \in \mathscr{H}, \boldsymbol{\Phi}$ be a Möbius transformation, and denote the power series coefficients of $f$ and $f \circ \Phi$ by $f_{k l}$ and $f_{k l}^{\prime}$, respectively. Because of (2.13)

$$
\|f\|^{2} \geq \delta^{2}[f, f]=\delta^{2}\left(b_{00}|f(0,0)|^{2}+\sum_{k, l=1}^{\infty}\left|f_{k l}\right|^{2} b_{k l}\right)
$$

and

$$
[f \circ \Phi, f \circ \Phi]=b_{00}|f(\Phi(0,0))|^{2}+\sum_{k, l=1}^{\infty}\left|f_{k l}^{\prime}\right|^{2} b_{k l}
$$

We have

$$
\begin{aligned}
\sum_{k, l=1}^{\infty}\left|f_{k l}^{\prime}\right|^{2} b_{k l} & \geq K_{1} \sum_{k, l=1}^{\infty}\left|f_{k l}\right|^{2} k l=K_{1}\|f \circ \Phi\|_{\mathscr{D}}^{2}=K_{1}\|f\|_{\mathscr{D}}^{2} \\
& =K_{1} \sum_{k, l=1}^{\infty}\left|f_{k l}\right|^{2} k l \geq \frac{K_{1}}{K_{2}} \sum_{k, l=1}^{\infty}\left|f_{k l}\right|^{2} b_{k l} .
\end{aligned}
$$


Hence

$$
\begin{aligned}
b_{00} \mid & \left.f(0,0)\right|^{2}+\sum_{k, l=1}^{\infty}\left|f_{k l}\right|^{2} b_{k l}=[f, f] \geq \delta^{8}[f \circ \Phi, f \circ \Phi] \\
& =\delta^{8}\left(b_{00}|f(\Phi(0,0))|^{2}+\sum_{k, l=1}^{\infty}\left|f_{k l}^{\prime}\right|^{2} b_{k l}\right) \\
& =\delta^{8}\left(b_{00}|f(\Phi(0,0))|^{2}+\frac{K_{1}}{K_{2}} \sum_{k, l=1}^{\infty}\left|f_{k l}\right|^{2} b_{k l}\right),
\end{aligned}
$$

from which we obtain

$$
b_{00}|f(0,0)|^{2}+\left(1-\delta^{8} \frac{K_{1}}{K_{2}}\right) \sum_{k, l=1}^{\infty}\left|f_{k l}\right|^{2} b_{k l} \geq \delta^{8} b_{00}|f(\Phi(0,0))|^{2} .
$$

The left hand side of this equation is finite since it is equivalent to $\|f\|^{2}$. Since $\Phi(0,0)$ can be any point in $\mathbb{U}^{2}$, it follows that every element of $\mathscr{H}$, i.e., of $\mathscr{D}\left(\mathbb{U}^{2}\right)$, is bounded. But a Dirichlet space contains unbounded elements. In $\mathbb{U}$, this is seen most easily by the Area Theorem; in $\mathbb{U}^{2}$, we take an unbounded function depending only on one variable. Therefore $b_{00}=0$ and we are done.

Corollary 2.3. Theorem B is true even if (1.3) holds only for $\Psi \in$ $\mathscr{M}^{*}$. Theorem A is true even if (1.2) holds only for $\Psi \in \mathscr{M}^{*}$.

Proof. The proof of Theorem B uses coordinate permutations nowhere. Theorem A is a consequence of Theorem B.

3. Boundary behavior. Dirichlet-type spaces. This section requires some new notions that were studied in $\mathbb{U}$ in [3] and [4]. For each $\delta=\left(\delta_{1}, \ldots, \delta_{n}\right)$ with each $0 \leq \delta_{j} \leq 1$, we define the Dirichlet-type spaces $\mathscr{D}_{\delta}\left(\mathbb{U}^{n}\right)$ to consist of those $f(z)=\sum_{\alpha} f_{\alpha} z^{\alpha} \in \mathscr{H}\left(\mathbb{U}^{n}\right)$ that satisfy

$$
\|f\|_{\mathscr{D}_{\delta}}=\sum_{\alpha} \alpha_{1}^{2 \delta_{1}} \cdots \alpha_{n}^{2 \delta_{n}}\left|f_{\alpha}\right|^{2}<\infty .
$$

This definition makes sense even if some $\delta_{j}=0$ if we interpret $0^{0}=$ 1. In fact, if all the $\delta_{j}=0$, then $\mathscr{D}_{\delta}\left(\mathbb{U}^{n}\right)=\mathscr{H}^{2}\left(\mathbb{U}^{n}\right)$. The space corresponding to $\delta_{1}=\cdots=\delta_{n}=1$ consists of functions $f$ with $D_{1} \cdots D_{n} f \in \mathscr{H}^{2}\left(\mathbb{U}^{n}\right)$. When $\delta_{1}=\cdots=\delta_{n}=1 / 2$, we have the Dirichlet space. For $n=1$, all Dirichlet-type spaces are contained in $\mathscr{H}^{2}(\mathbb{U})$, but this is not true if $n>1$. 
Some subclasses of $\mathscr{D}_{\delta}\left(\mathbb{U}^{n}\right)$ have certain integral representations: If $F \in \mathscr{L}^{2}\left(\mathbb{T}^{n}\right)$, for $0 \leq \delta_{j}<1$ and $z \in \mathbb{U}^{n}$, set

$$
f(z)=\int_{\mathbb{T}^{n}} F(\zeta) \prod_{j=1}^{n} \frac{1}{\left(1-z_{j} \bar{\zeta}_{j}\right)^{1-\delta_{j}}} d \lambda_{n}(\zeta) .
$$

The product is the Cauchy kernel each of whose factors is raised to a fractional power. Omitting $j$, each factor can be expanded as

$$
(1-z \bar{\zeta})^{\delta-1}=\sum_{\alpha=0}^{\infty} b_{\alpha} z^{\alpha \bar{\zeta}^{\alpha}}
$$

where

$$
b_{\alpha}=\frac{\Gamma(1-\delta+\alpha)}{\Gamma(1-\delta) \Gamma(1+\alpha)} \sim \frac{1}{\alpha^{\delta}} .
$$

In particular $b_{0}=1$. Let $c_{\alpha}$ be the $\left(\alpha_{1}, \ldots, \alpha_{n}\right)$ th Fourier coefficient of $F$; i.e.,

$$
c_{\alpha}=\int_{\mathbb{T}^{n}} \bar{\zeta}^{\alpha} F(\zeta) d \lambda_{n}(\zeta)
$$

Setting $f_{\alpha}=b_{\alpha_{1}} \cdots b_{\alpha_{n}} c_{\alpha}$, we get

$$
\begin{aligned}
f(z) & =\int_{\mathbb{T}^{n}} \prod_{j=1}^{n} \sum_{\alpha_{j}=0}^{\infty} b_{\alpha_{j}} z_{j}^{\alpha_{j}} \bar{\zeta}_{j}^{\alpha_{j}} F(\zeta) d \lambda_{n}(\zeta) \\
& =\sum_{\alpha} b_{\alpha_{1}} \cdots b_{\alpha_{n}} z^{\alpha} \int_{\mathbb{T}^{n}} \bar{\zeta}^{\alpha} F(\zeta) d \lambda_{n}(\zeta) \\
& =\sum_{\alpha} b_{\alpha_{1}} \cdots b_{\alpha_{n}} z^{\alpha} c_{\alpha}=\sum_{\alpha} f_{\alpha} z^{\alpha} .
\end{aligned}
$$

Now

$$
\|f\|_{\mathscr{D}_{\delta}}=\sum_{\alpha} \alpha_{1}^{2 \delta_{1}} \cdots \alpha_{n}^{2 \delta_{n}}\left|b_{\alpha_{1}}\right|^{2} \cdots\left|b_{\alpha_{n}}\right|^{2}\left|c_{\alpha}\right|^{2} \sim \sum_{\alpha}\left|c_{\alpha}\right|^{2}<\infty .
$$

Hence $f \in \mathscr{D}_{\delta}\left(\mathbb{U}^{n}\right)$; i.e., any $f$ given by (3.1) is in a Dirichlet-type space.

But not all $f \in \mathscr{D}_{\delta}\left(\mathbb{U}^{n}\right)$ have integral representations as in (3.1), because a Dirichlet-type space does not control all the power series coefficients of its members. However, we can define a space $\tilde{\mathscr{D}}_{\delta}\left(\mathbb{U}^{n}\right)$ similar to $\tilde{\mathscr{D}}\left(\mathbb{U}^{n}\right)$ in which an integral representation is possible. Let's concentrate on the case $n=2$ again for simplicity. With obvious 
notation, $f \in \tilde{\mathscr{D}}_{\delta}\left(\mathbb{U}^{2}\right)$ if and only if

$$
\begin{aligned}
\|f\|_{\tilde{\mathscr{D}}_{\delta}}^{2}= & \left|f_{00}\right|^{2}+\sum_{k=1}^{\infty} k^{2 \delta_{1}}\left|f_{k 0}\right|^{2}+\sum_{l=1}^{\infty} l^{2 \delta_{2}}\left|f_{0 l}\right|^{2} \\
& +\sum_{k, l=1}^{\infty} k^{2 \delta_{1}} l^{2 \delta_{2}}\left|f_{k l}\right|^{2}<\infty
\end{aligned}
$$

Given $f(z)=\sum_{k l} f_{k l} z^{k} w^{l} \in \tilde{\mathscr{D}}_{\delta}\left(\mathbb{U}^{2}\right)$, let $c_{k l}=f_{k l} / b_{k} b_{l}$ if $(k, l) \in$ $\mathbb{N}^{2}$ (recall that $b_{0}=1$ ), and let $c_{k l}=0$ otherwise. Then, using (3.2),

$$
\sum_{(k, l) \in \mathbb{Z}^{2}}\left|c_{k l}\right|^{2}=\left|c_{00}\right|^{2}+\sum_{k=1}^{\infty}\left|c_{k 0}\right|^{2}+\sum_{l=1}^{\infty}\left|c_{0 l}\right|^{2}+\sum_{k, l=1}^{\infty}\left|c_{k l}\right|^{2} \sim\|f\|_{\tilde{D}_{\delta}}^{2}<\infty .
$$

Thus there is an $F \in \mathscr{L}^{2}\left(\mathbb{T}^{2}\right)$ such that $\hat{F}(k, l)=c_{k l}$. Therefore

$$
\begin{aligned}
f(z) & =\sum_{k, l=0}^{\infty} f_{k l} z^{k} w^{l}=\sum_{k, l=0}^{\infty} c_{k l} b_{k} b_{l} z^{k} w^{l} \\
& =\sum_{k, l=0}^{\infty} b_{k} b_{l} z^{k} w^{l} \int_{\mathbb{T}^{2}} \bar{\zeta}_{1}^{k} \bar{\zeta}_{2}^{l} F(\zeta) d \lambda_{2}(\zeta) \\
& =\int_{\mathbb{T}^{2}} \frac{F(\zeta) d \lambda_{2}(\zeta)}{\left(1-\bar{\zeta}_{1} z\right)^{1-\delta_{1}\left(1-\bar{\zeta}_{2} w\right)^{1-\delta_{2}}}} .
\end{aligned}
$$

Clearly $F$ is not unique. In fact, $c_{k l}$ can be defined arbitrarily for $(k, l) \notin \mathbb{N}^{2}$ as long as we retain $\sum_{(k, l) \in \mathbb{Z}^{2}}\left|c_{k l}\right|^{2}<\infty$.

Kernels and potentials. From now on, we will also use $e^{i \theta}$ for $\zeta_{j} \in \mathbb{T}, e^{i \varphi_{j}}$ for $\eta_{j} \in \mathbb{T}$, and $r_{j} e^{i \theta_{j}}=r_{j} \zeta_{j}$ for $z_{j} \in \mathbb{U}$. The point $(1, \ldots, 1) \in \mathbb{T}^{n}$ corresponding to $\theta_{1}=\cdots=\theta_{n}=0$ will act like the origin in $\mathbb{R}^{n}$. Now the Poisson kernel takes the more familiar form

$$
P_{r}(\theta)=\prod_{j=1}^{n} \frac{1-r_{j}^{2}}{1-2 r_{j} \cos \theta_{j}+r_{j}^{2}},
$$

and it is considered as a function of $\theta$ indexed by $r$. So the $\mathscr{L}^{p}$ norm of a Poisson integral will be obtained by an integration on the $\theta$-variable and will still depend on $r$.

A kernel $K$ is a nonnegative $\mathscr{L}^{1}$-function on $\mathbb{T}^{n}$ which is even and decreasing in each $\left|\theta_{j}\right|$ when the other variables are kept fixed. We will also have $K(1, \ldots, 1)=\infty$ and normalize as $\|K\|_{1}=1$. A potential is the convolution of an $\mathscr{L}^{p}$-function $F$ on $\mathbb{T}^{n}$ with a kernel. Thus (3.1) defines $f(z) \in \tilde{\mathscr{D}}_{\delta}\left(\mathbb{U}^{n}\right)$ as a potential. The Poisson integral is simply the convolution with the Poisson kernel. 
Let's define the Bessel kernels on the torus. For $0<\delta_{j} \leq 1$, let

$$
\begin{aligned}
G_{\delta}(\zeta)=\prod_{j=1}^{n} g_{\delta_{j}}\left(\theta_{j}\right) & =\prod_{j=1}^{n}\left(1+\frac{1}{2} \sum_{\alpha_{j} \neq 0}\left|\alpha_{j}\right|^{-\delta_{j} \zeta_{j}^{\alpha_{j}}}\right) \\
& =\prod_{j=1}^{n}\left(1+\sum_{\alpha_{j}=1}^{\infty} \alpha_{j}^{-\delta_{j}} \cos \left(\alpha_{j} \theta_{j}\right)\right)
\end{aligned}
$$

where each $g_{\delta_{j}}$ is a Bessel kernel on the unit circle. $g_{\delta}(0)=\infty$, $g_{\delta}$ is a decreasing function of $|\omega|$ for $\omega \neq 0, g_{\delta}(\omega)>0$, and $g_{\delta}(-\omega)=g_{\delta}(\omega)$. Each $g_{\delta_{j}} \in \mathscr{L}^{1}(\mathbb{T})$, so $G_{\delta} \in \mathscr{L}^{1}\left(\mathbb{T}^{n}\right)$, and $\left\|G_{\delta}\right\|_{1}=\prod_{j=1}^{n}\left\|g_{\delta_{j}}\right\|_{1}=1$. When $0<\delta<1$,

$$
g_{\delta}(\omega) \approx\left|\sin \frac{\omega}{2}\right|^{\delta-1} \quad \text { as } \omega \rightarrow 0 \text {. }
$$

Also $g_{1}(\omega)=1-\log |2 \sin (\omega / 2)| . \quad P_{r}\left[g_{\delta}\right]=P_{r} * g_{\delta}$ is the harmonic extension of $g_{\delta}$ to $\mathbb{U}$. As $r \rightarrow 1$, it satisfies the following:

$$
\begin{aligned}
\left\|P_{r} * g_{\delta}\right\|_{q} \sim(1-r)^{\delta-1 / p} & (\delta p<1), \\
\left\|P_{r} * g_{\delta}\right\|_{q} \sim\left(\log \frac{1}{1-r}\right)^{1 / q} & (\delta p=1, p>1), \\
\left\|P_{r} * g_{1}\right\|_{\infty} \sim \log \frac{1}{1-r} & (\delta=1, p=1) .
\end{aligned}
$$

$P_{r}\left[G_{\delta}\right]$ possesses these properties in each variable seperately.

On the unit circle, for $0<\delta<1$, the modified Bessel kernels are

$$
\tilde{g}_{\delta}(\omega)=\left(1-e^{i \omega}\right)^{\delta-1} \text { and } \quad \tilde{g}_{1}(\omega)=\log \frac{1}{1-e^{i \omega}} .
$$

On the torus, let $\tilde{G}_{\delta}(\zeta)=\prod_{j=1}^{n} \tilde{g}_{\delta_{j}}\left(\theta_{j}\right)$. These functions are not positive, so they are not properly kernels, but they are dominated by the Bessel kernels: There are constants $C_{\delta}>0$ such that $\left|\tilde{G}_{\delta}\right| \leq$ $C_{\delta} G_{\delta}$. If each $\delta_{j}$ is less than 1 ,

$$
P_{r}\left[\tilde{G}_{\delta}\right](\theta)=\prod_{j=1}^{n} P_{r_{j}}\left[\tilde{g}_{\delta}\right]\left(\theta_{j}\right)=\prod_{j=1}^{n}\left(1-z_{j}\right)^{\delta_{j}-1},
$$

with a logarithmic term if some $\delta_{k}=1$. For $F \in \mathscr{L}^{p}\left(\mathbb{T}^{n}\right)$, the map that takes $F$ to $G_{\delta} * F$ is one-to-one, and the Cauchy integral of $\tilde{G}_{\delta} * F$ is the same as its Poisson integral:

$$
\begin{aligned}
P_{r}\left[\tilde{G}_{\delta} * F\right](\theta) & =\left(P_{r} * \tilde{G}_{\delta} * F\right)(\theta) \\
& =\int_{\mathbb{T}^{n}} F(\zeta) \prod_{j=1}^{n} \frac{1}{\left(1-z_{j} \bar{\zeta}_{j}\right)^{1-\delta,}} d \lambda_{n}(\zeta)=f(z) .
\end{aligned}
$$


Thus we have obtained the integral in (3.1). From now on, $F$ and $f$ will always be related as in this equation. $\delta_{j}=1$ does not give rise to a Cauchy-type integral; so we will not pay any attention to this case any more.

Tangential limits. Define the tangential approach regions to the unit circle:

$$
\begin{aligned}
& A_{\gamma, c}(\varphi)=\left\{r e^{i \theta}: 1-r>c\left|\sin \frac{\theta-\varphi}{2}\right|^{\gamma}\right\} \\
& E_{\gamma, c}(\varphi)=\left\{r e^{i \theta}: 1-r>\exp \left(-c\left|\sin \frac{\theta-\varphi}{2}\right|^{-\gamma}\right)\right\} .
\end{aligned}
$$

$A_{\gamma, c}(\varphi)$ has (polynomial) order of contact $\gamma$, and $E_{\gamma, c}(\varphi)$ makes exponential contact, with $\mathbb{T}$. A function $f$ defined in $\mathbb{U}$ has $A_{\gamma}\left(E_{\gamma}\right)$ limit $L$ at $e^{i \varphi}$ if $f(z) \rightarrow L$ as $z \rightarrow e^{i \varphi}$ within $A_{\gamma, c}\left(E_{\gamma, c}\right)$ for every $c>0$. In [3], it was shown that Poisson integrals of the modified Bessel potentials have $A_{\gamma}$-limits a.e. on $\mathbb{T}$ if $\delta p<1$ for $\gamma=\frac{1}{1-\delta p}$, and $E_{\gamma}$-limits a.e. on $\mathbb{T}$ if $\delta p=1$ for $\gamma=q-1$.

Let $Q=Q(\eta, s)$ be the cube centered at $\eta \in \mathbb{T}^{n}$ with sides $s=$ $\left(s_{1}, \ldots, s_{n}\right)$, where each $s_{j}$ has the same order as $\max \left\{s_{j}: 1 \leq\right.$ $j \leq n\} \rightarrow 0$. Its volume is $\lambda_{n}(Q)=s_{1} \cdots s_{n}$. If $F \in \mathscr{L}^{p}\left(\mathbb{T}^{n}\right)$, its Hardy-Littlewood maximal function is

$$
\left(M_{p} F\right)(\eta)=\sup _{0<s_{1}, \ldots, s_{n} \leq 1}\left(\frac{1}{\lambda_{n}(Q)} \int_{Q}|f|^{p} d \lambda_{n}\right)^{1 / p} .
$$

$M_{1}$ is of weak type $(1,1)$; and since $M_{p} F=\left(M_{1}|F|^{p}\right)^{1 / p}, M_{p}$ is of weak type $(p, p)$. Thus there are $C_{p}$ such that

$$
\lambda_{n}\left(\left\{M_{p} F>t\right\}\right) \leq \frac{C_{p}}{t^{p}}\|F\|_{p}^{p} \quad\left(F \in \mathscr{L}^{p}\left(\mathbb{T}^{n}\right), t \in(0, \infty)\right) .
$$

The proofs of the following assertions are similar to the proofs given in [3] for $n=1$ and will be omitted. Some of them are valid in more general situations. The first result is obtained using the straightforward inequality

$$
\int_{\mathbb{T}^{n}}|F| G_{\delta} d \lambda_{n} \leq\left(M_{1} F\right)(1, \ldots, 1) \int_{\mathbb{T}^{n}} G_{\delta} d \lambda_{n}=\left(M_{1} F\right)(1, \ldots, 1),
$$

which holds for any $F \in \mathscr{L}^{1}\left(\mathbb{T}^{n}\right)$, and whose proof is also in [3].

THeorem 3.1. There is a $C_{p}<\infty$ such that for $F \in \mathscr{L}^{p}\left(\mathbb{T}^{n}\right)$ and $\zeta, \eta \in \mathbb{T}^{n}$,

$$
\left|\left(G_{\delta} * F\right)(\zeta)\right| \leq C_{p}\left[\left(M_{p} F\right)(\eta)\left(\prod_{j=1}^{n}\left|\zeta_{j}-\eta_{j}\right|^{1 / p}\right)\left\|G_{\delta}\right\|_{q}+\left(M_{1} F\right)(\eta)\right] .
$$


Convolution of two kernels is a kernel; so Theorem 3.1 holds with $P_{r} * G_{\delta}=P_{r}\left[G_{\delta}\right]$ in place of $G_{\delta}$, which has the desired properties $\left\|P_{r} * G_{\delta}\right\|_{1}=\left\|P_{r}\right\|_{1}\left\|G_{\delta}\right\|_{1}=1$ and $\left\|P_{r} * G_{\delta}\right\|_{q}=\prod_{j=1}^{n}\left\|P_{r_{j}} * g_{\delta_{j}}\right\|_{q}$. The Hölder inequality gives $M_{1} F \leq M_{p} F$. In addition, $\left|\zeta_{j}-\eta_{j}\right|$ can be replaced by $\left|\theta_{j}-\varphi_{j}\right|$, or even by $\left|\sin \left(\left(\theta_{j}-\varphi_{j}\right) / 2\right)\right|$, since they are all of the same order as $\zeta \rightarrow \eta$. Lastly, we can put $\tilde{G}_{\delta}$ in place of $G_{\delta}$ on the left side of the inequality since the latter dominates the former. Hence Theorem 3.1 yields

TheOREM 3.2. If $F \in \mathscr{L}^{p}\left(\mathbb{T}^{n}\right)$, then, using $z=r e^{i \theta}$, for all $e^{i \theta}, e^{i \varphi}$ $\in \mathbb{T}^{n}$,

$$
|f(z)| \leq C_{p}\left(M_{p} F\right)(\varphi)\left[1+\prod_{j=1}^{n}\left|\sin \frac{\theta_{j}-\varphi_{j}}{2}\right|^{1 / p}\left\|P_{r_{j}} * g_{\delta_{j}}\right\|_{q}\right] .
$$

For given $\varphi$, any bound on the product on the right side gives a bound on $f(z)$. This leads us to the tangential approach regions to $\mathbb{T}^{n}$. So fix an $\eta \in \mathbb{T}^{n}$. As $z \rightarrow \zeta$, all $r_{j} \rightarrow 1$; and because of (3.3) and (3.4), $\left\|P_{r} * G_{\delta}\right\|_{q} \sim \prod_{j=1}^{n} b_{j}$, where $b_{j}=\left(1-r_{j}\right)^{\delta_{J}-1 / p}$ or $b_{j}=\left(\log \left(1 /\left(1-r_{j}\right)\right)\right)^{1 / q}$ depending on whether $\delta_{j} p<1$ or $\delta_{j} p=1$, respectively. In other words, an approach region should be determined by

$$
\prod_{j=1}^{n} b_{j}\left|\sin \frac{\theta_{j}-\varphi_{j}}{2}\right|^{1 / p}<c
$$

So define $B_{\gamma, c}(\eta)$ by

$$
B_{\gamma, c}(\eta)=B_{\gamma, c}(\varphi)=\left\{z \in \mathbb{U}^{n}: \prod_{j=1}^{n} b_{j}^{-1 / \gamma_{j}}\left|\sin \frac{\theta_{j}-\varphi_{j}}{2}\right|<c\right\} .
$$

Each of the factors in the above product is related to one of the regions in (3.5). In particular, points in a cartesian product of onedimensional approach regions such as $B_{\gamma_{1}, c_{1}}\left(\varphi_{1}\right) \times \cdots \times B_{\gamma_{n}}, c_{n}\left(\varphi_{n}\right)$, where each $B_{\gamma_{j}, c_{j}}\left(\varphi_{j}\right)$ is either $A_{\gamma_{j}, c_{j}}\left(\varphi_{j}\right)$ or $E_{\gamma_{j}, c_{j}}\left(\varphi_{j}\right)$, satisfy the criterion for being in $B_{\gamma, c}(\eta)$. Hence an approach region can make exponential contact with $\mathbb{T}^{n}$ in one (complex) direction and polynomial contact in another. For $\eta \in \mathbb{T}^{n}$, the maximal functions associated to these approach regions are defined as

$$
\left(M_{G_{\delta}, \gamma, c} f\right)(\eta)=\sup \left\{|f(z)|: z \in B_{\gamma, c}(\eta)\right\} .
$$

A function $f$ defined in $\mathbb{U}^{n}$ is said to have $B_{\gamma}$-limit $L$ at $\eta \in \mathbb{T}^{n}$ if $f(z) \rightarrow L$ as $z \rightarrow \eta$ within $B_{\gamma, c}(\eta)$ for every $c>0$. 
Theorem 3.3. If $F \in \mathscr{L}^{p}\left(\mathbb{T}^{n}\right)$ and $t \in(0, \infty)$, there is a $C=$ $C(p, c)$ such that

$$
\lambda_{n}\left(\left\{\boldsymbol{M}_{G_{\delta}, \gamma, c} f>t\right\}\right)<\frac{C}{t^{p}}\|F\|_{p}^{p},
$$

where

$$
\gamma_{j}=\frac{1}{1-\delta_{j} p} \quad \text { if } \delta_{j} p<1 \quad \text { and } \quad \gamma_{j}=q-1 \quad \text { if } \delta_{j} p=1 .
$$

This follows from the weak-type- $(p, p)$ estimate for $M_{p}$. The weak-type estimate gives rise to a convergence theorem via classical arguments. This is the content of part (i) of Theorem C. The case $p=1$ of part (ii) of that theorem also follows from Theorem 3.3. Henceforth, $p, \delta$, and $\gamma$ will always be related by (3.8).

Capacities. For $E \subset \mathbb{T}^{n}$, let $T\left(G_{\delta}, p, E\right)$ be the set of all nonnegative $F \in \mathscr{L}^{p}\left(\mathbb{T}^{n}\right)$ such that $\left(G_{\delta} * F\right)(\zeta) \geq 1$ for all $\zeta \in E$. The p-capacity of $E$ is

$$
\Sigma_{G_{\delta}}(E)=\inf \left\{\|F\|_{p}^{p}: F \in T\left(G_{\delta}, p, E\right)\right\} .
$$

$\Sigma_{G_{\delta}}(E)=0$ implies $\lambda_{n}(E)=0$. The functions $G_{\delta} * F$ are defined $\Sigma_{G_{\delta}}$-almost everywhere. If $F \in T\left(P_{r} * G_{\delta}, p, E\right)$, then $\Sigma_{P_{r}}(E) \leq$ $\Sigma_{P_{r} * G_{\delta}}(E)$. For $\eta \in \mathbb{T}^{n}$ and fixed $\rho>1$, let

$$
\Gamma(\eta)=\left\{z \in \mathbb{U}^{n}:\left|z_{j}-\eta_{j}\right|<\rho\left(1-\left|z_{j}\right|\right), 1 \leq j \leq n\right\},
$$

and set $S(E)=\mathbb{U}^{n} \backslash \bigcup_{\eta \notin E} \Gamma(\eta) . \Gamma(\eta)$ is the cartesian product of $n$ sets each of which is asymptotic, as $z_{j} \rightarrow \eta_{j}$, to an angle-shaped approach region in $\mathbb{U}$ with vertex at $\eta_{j}$. For $\eta \in \mathbb{T}^{n}$, the nontangential maximal function is

$$
(N f)(\eta)=\sup \{|f(z)|: z \in \Gamma(\eta)\} .
$$

For $W \subset \mathbb{U}^{n}, J_{c}^{\gamma}(W)$ is the set of $\eta \in \mathbb{T}^{n}$ for which $W$ intersects $B_{\gamma, c}(\eta)$.

LEMMA 3.4. There exists a constant $b=b(n)>0$ such that if $F \geq 0$ on $\mathbb{T}^{n}, F \geq 1$ on $E \in \mathbb{T}^{n}$, and $z \in S(E)$, then $P_{r}[F](z)>b$.

We will use this lemma with $G_{\delta} * F$ in place of $F$. It leads to the following lower estimate for capacities. 
Proposition 3.5. If $F \in \mathscr{L}^{1}\left(\mathbb{T}^{n}\right)$ and $0<t<\infty$, then $\left\{M_{G_{\delta}, \gamma, c} f\right.$ $>t\}$ is contained in $J_{c}^{\gamma}(S(\{N f>t\}))$. Thus, there is a $C=C(p, c)$ such that if $t \in(0, \infty)$ and $F \in \mathscr{L}^{1}\left(\mathbb{T}^{n}\right)$, we have

$$
\lambda_{n}\left(\left\{M_{G_{\delta}, \gamma, c} f>t\right\}\right) \leq C \Sigma_{G_{\delta}}(\{N f>t\}) .
$$

THEOREM 3.6. For $1<p<\infty$, there is a constant $C_{p}<\infty$ such that if $F \in \mathscr{L}^{p}\left(\mathbb{T}^{n}\right)$ and $F \geq 0$, then

$$
\int_{0}^{\infty} \Sigma_{G_{\delta}}\left(\left\{G_{\delta} * F>t\right\}\right) d\left(t^{n}\right) \leq C_{p}\|F\|_{p}^{p}
$$

Combining Lemma 3.4, Proposition 3.5, and Theorem 3.6 with the fact that $G_{\delta}$ dominates $\tilde{G}_{\delta}$ and that $N\left(G_{\delta} * F\right)=G_{\delta} * N F$, we obtain the strong-type estimates in part (ii) of Theorem $\mathrm{C}$.

Theorem C. Let $1 \leq p<\infty, F \in \mathscr{L}^{p}\left(\mathbb{T}^{n}\right), 0<\delta_{j}<1$, define $f$ as in (3.1), pick $\gamma_{j}$ as in (3.8), and for $\zeta \in \mathbb{T}^{n}$, construct $B_{\gamma, c}(\zeta)$ as in (3.7).

(i) The $B_{\gamma}$-limit of $f$ exists a.e. $\left[\lambda_{n}\right]$ on $\mathbb{T}^{n}$

(ii) There are positive constants $C_{p}$ such that

$$
\begin{aligned}
\left\|M_{G_{\delta}, \gamma, c} f\right\|_{p} & \leq C_{p}\|F\|_{p} & & (1<p<\infty), \\
\lambda_{n}\left(\left\{M_{g_{\delta}, \gamma, c} f>t\right\}\right) & \leq \frac{C_{1}}{t}\|F\|_{1} & & (p=1,0<t<\infty) .
\end{aligned}
$$

If $\zeta \in \partial \mathbb{U}^{n} \backslash \mathbb{T}^{n}$, then only one component of $\zeta$, say the $n$ th, has $\left|\zeta_{n}\right|=1$. Then the first $n-1$ factors in the product in (3.6) are bounded as $z \rightarrow \zeta$. So in this case, it suffices to apply the one-variable result in the $n$th variable. The approach regions are restricted only in the $n$th component as in $(3.5)$, and $\left(z_{1}, \ldots, z_{n-1}\right)$ can approach $\left(\zeta_{1}, \ldots, \zeta_{n-1}\right) \in \partial \mathbb{U}^{n}$ in any manner whatsoever. Theorem $\mathrm{C}$ remains valid except that in part (ii), we would use one-dimensional norm and Lebesgue measure.

When $p=2$ and all the $\delta_{j}=1 / 2$, this theorem takes care of $\tilde{\mathscr{D}}\left(\mathbb{U}^{n}\right)$, but cannot deal with $\mathscr{D}\left(\mathbb{U}^{n}\right)$. Thus the functions in the modified Dirichlet space have tangential $\left(B_{\left.(1, \ldots, 1)^{-}\right)}\right.$limits at almost every boundary point of the unit polydisc. When $n=1$, since $\mathscr{H}^{2}(\mathbb{U})$ includes all Dirichlet-type spaces, elements of $\mathscr{D}(\mathbb{U})$ have nontangential limits a.e. on $\mathbb{T}$. 
Now we will look at the size of the exceptional sets. From Lemma 3.4, part (ii) of Theorem C, and the first part of Proposition 3.5, we obtain

LEMMA 3.7. If $1<p<\infty$, then for some $C=C(p, c)$

$$
\Sigma_{P_{r}}\left(J_{c}^{\gamma}(S(E))\right) \leq C \Sigma_{P_{r} * G_{\delta}}(E) .
$$

Hence for $F \in \mathscr{L}^{p}\left(\mathbb{T}^{n}\right)$,

$$
\Sigma_{P_{r}}\left(\left\{M_{G_{\delta}, \gamma, c} f>t\right\}\right) \leq C \Sigma_{P_{r} * G_{\delta}}(\{N f>t\}) .
$$

TheOREM 3.8. If $1<p<\infty$ and $F \in \mathscr{L}^{p}\left(\mathbb{T}^{n}\right)$, then

$$
\int_{0}^{\infty} \Sigma_{P_{r}}\left(\left\{M_{G_{\delta}, \gamma, c} f>t\right\}\right) d\left(t^{n}\right) \leq C\|F\|_{p}^{p},
$$

and thus

$$
\Sigma_{P_{r}}\left(\left\{M_{G_{\delta}, \gamma, c} f>t\right\}\right) \leq C \frac{\|F\|_{p}^{p}}{t^{p}} .
$$

This theorem is an analog of Theorem $\mathrm{C}$ in the language of capacities and proved similarly.

TheOREM D. Let $1<p<\infty, F \in \mathscr{L}^{p}\left(\mathbb{T}^{n}\right)$, and $f$ be as in (3.1).

(i) There is a set $E_{1} \subset \mathbb{T}^{n}$ with $\Sigma_{P_{r} * G_{\delta}}\left(E_{1}\right)=0$ such that the nontangential limit of $f$ exists at every point of $\mathbb{T}^{n} \backslash E_{1}$.

(ii) There is a set $E_{2} \subset \mathbb{T}^{n}$ with $\Sigma_{P_{r}}\left(E_{2}\right)=0$ such that the $B_{\gamma}$-limit of $f$ exists at every point of $\mathbb{T}^{n} \backslash E_{2}$.

This result is a consequence of the basic properties of capacities and Theorem 3.10. For points on $\partial \mathbb{U}^{n} \backslash \mathbb{T}^{n}$, the one-variable result can again be used to reach a similar conclusion. Hence if $p=2$ and all the $\delta_{j}=1 / 2$, the points on $\partial \mathbb{U}^{n}$ where the modified Dirichlet space does not have nontangential limits have zero capacity in some sense.

\section{REFERENCES}

[1] J. Arazy and S. D. Fisher, The uniqueness of the Dirichlet space among Möbiusinvariant Hilbert spaces, Illinois J. Math., 29 (1985), 449-462.

[2] E. Hewitt and K. Ross, Abstract Harmonic Analysis, I, 2nd ed., Grundlehren Math. Wiss., vol. 115, Springer, Berlin, 1979.

[3] A. Nagel, W. Rudin and J. H. Shapiro, Tangential boundary behavior of functions in Dirichlet-type spaces, Ann. of Math., 116 (1982), 331-360.

[4] W. Rudin, The variation of holomorphic functions on tangential boundary curves, Michigan Math. J., 32 (1985), 41-45. 
[5] K. Zhu, Möbius-invariant Hilbert spaces of holomorphic functions in the unit ball of $\mathbb{C}^{n}$, Trans. Amer. Math. Soc., 323 (1991), 823-842.

Received May 11, 1992. Part of this work represents a part of the author's Ph.D. thesis completed under the supervision of Walter Rudin.The author wishes to thank Walter Rudin and Frank Forelli for several useful discussions. Supported under TBAG-ÇG2 by TÜB.ITAK.

Mathematics Department

UNIVERSITY OF WISCONSIN

MADISON, WI 53706

Current address: Mathematics Department

Middle East Technical University

Ankara 06531 Turkey

E-mail address: mathtk@vm.cc.metu.edu.tr 


\section{CONTENTS}

D. Bisch, A note on intermediate subfactors , . , . . . . . . . . . . . . . . . . 201

M. J. Carro and J. Soria, Tent spaces over general approach regions and pointwise

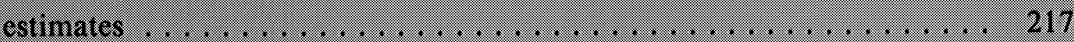

J. A. Charris, M. E. H. Ismail, and S. Monsalve, On sieved orthogonal polynomials X: general blocks of recurrence relations . . . . . . . . . . . . . . . . 237

K. S. Chou and T. Y. H. Wan, Asymptotic radial symmetry for solutions of $\Delta u+$ $e^{\prime \prime}=0$ in a punctured disc . . . . . . . . . . . . . . . . . . . . . . . 269

M. E. Fogel, Knots with algebraic unknotting number one . . . . . . . . . . . . . 277

K. N. Jones, The structure of closed nonpositively curved Euclidean cone 3-manifolds . . . . . . . . . . . . . . . . . . . . . . . . . . . . . . . . 297

M. Kaneda, On the Frobenius morphism of flag schemes . . . . . . . . . . 315

H. T. Kaptanoğlu, Möbius-invariant Hilbert spaces in polydiscs . . . . . . . . . 337

K. Kuribayashi, The cohomology ring of the spaces of loops on Lie groups and homogeneous spaces ............................ 361

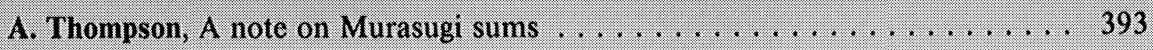




\title{
PACIFIC JOURNAL OF MATHEMATICS
}

\author{
Volume $163 \quad$ No. $2 \quad$ April 1994
}

A note on intermediate subfactors

201

DIETMAR BISCH

Tent spaces over general approach regions and pointwise estimates

217

MARÍA J. CARRO and JAVIER SORIA

On sieved orthogonal polynomials. X. General blocks of recurrence

237 relations

JAIro A. Charris, Mourad ISMAIL and SERGio Monsalve

Asymptotic radial symmetry for solutions of $\Delta u+e^{u}=0$ in a punctured disc

Kai Seng (Kaising) Chou (Tso) and Tom YaU-Heng Wan

Knots with algebraic unknotting number one

MicAh Elton Fogel

The structure of closed non-positively curved Euclidean cone

3-manifolds

KERRY NELSON JONES

On the Frobenius morphism of flag schemes

MASAHARU KANEDA

Möbius-invariant Hilbert spaces in polydiscs

H. TuRgay KaptanOGLU

The cohomology ring of the spaces of loops on Lie groups and homogeneous spaces

KATSUHIKO KURIBAYASHI

A note on Murasugi sums

ABIGAIL A. THOMPSON 